\title{
Parâmetros usados na eletrofototerapia para a cicatrização de feridas: Uma revisão
}

\section{de escopo}

\author{
Parameters used in electrophototherapy for wound healing: A scope review \\ Parámetros utilizados en electrofototerapia para la cicatrización de heridas: Una revisión del
}

alcance

Recebido: 17/05/2021 | Revisado: 11/05/2021 | Aceito: 19/05/2021 | Publicado: 06/06/2021

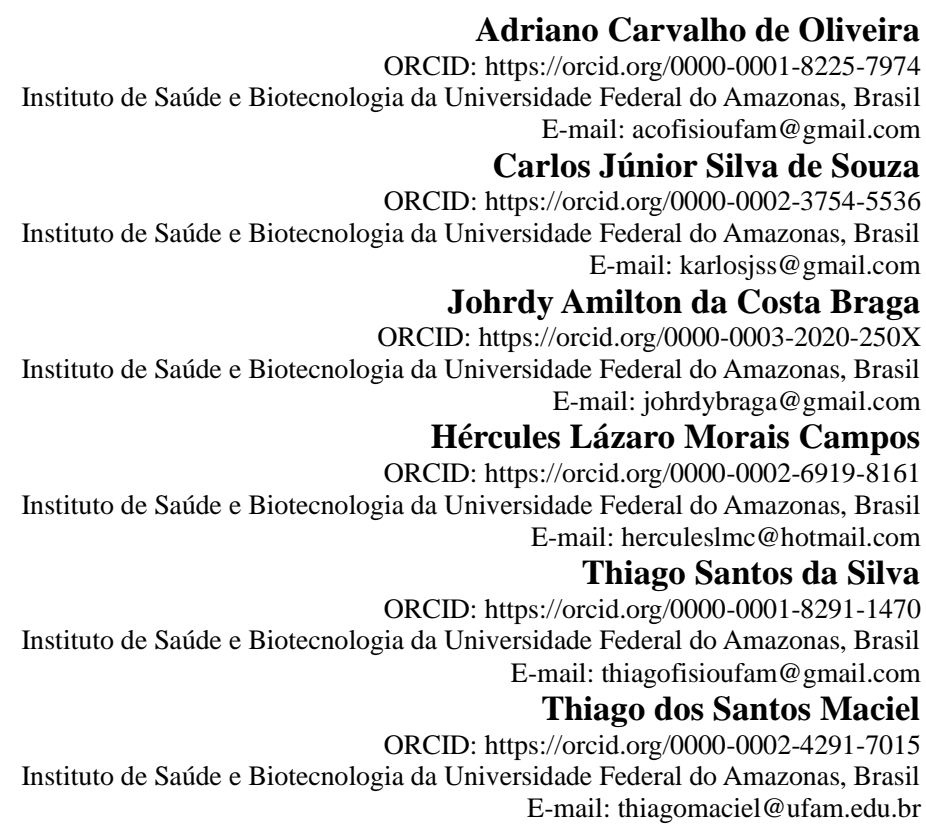

\section{Resumo}

Objetivo: Apontar parâmetros utilizados para a cicatrização de feridas através de modalidades eletrofototerapêuticas em pacientes com úlceras por pressão e diabéticas. Métodos: Buscou-se artigos nas bases de dados: PubMed, BVS, SciELO e PEDro, publicadas entre 2010 e maio de 2020. Dois autores analisaram de forma independente os critérios de elegibilidade através da Escala PEDro. Resultados: Os estudos incluídos apontam que os recursos eletrofototerapêuticos podem ser eficazes no tratamento das úlceras por pressão e diabéticas e que esses recursos aplicados em conjunto com a intervenção convencional otimizam a melhora do paciente. Conclusões: Verificou-se uma grande heterogeneidade nos parâmetros analisados, sendo os mais eficazes a Luz de espectro, com 400 à $800 \mathrm{~nm}$, $0,18 \mathrm{~W} / \mathrm{cm}^{2}, 4$ minutos, 2 aplicações por dia, durante 12 semanas. O LED, com $625,660,850 \mathrm{~nm}, 2,4 \mathrm{~J} / \mathrm{cm} 2,50 \%, 5$ minutos, 3 aplicações por semana, durante 8 semanas. A LLLT, com $685 \mathrm{~nm}, 0,05 \mathrm{~W} / \mathrm{cm}^{2}, 10 \mathrm{~J} / \mathrm{cm} 2,3$ minutos, 6 aplicações por semana, com duração de 2 à 20 semanas. E a CAV, com 100 pps, 0,24 ou 0,25 A, $154 \mu$ s, $100 \mathrm{~V}, 250$ ou $360 \mu \mathrm{C} / \mathrm{s}, 50$ minutos, 5 aplicações por semana, com duração de 6 ou 8 semanas.

Palavras-chave: Cicatrização; Fototerapia; Lesão por pressão; Pé diabético; Terapia por estimulação elétrica.

\footnotetext{
Abstract

Objective: Point out parameters used for wound healing through electrophototherapeutic modalities in patients with pressure ulcers and diabetics. Methods: We searched for articles in the databases: PubMed, BVS, SciELO and PEDro, published between 2010 and May 2020. Two authors independently analyzed the eligibility criteria using the PEDro Scale. Results: The included studies indicate that electrophototherapeutic resources can be effective in the treatment of pressure and diabetic ulcers and that these resources applied in conjunction with conventional intervention optimize patient improvement. Conclusions: There was a great heterogeneity in the analyzed parameters, the most efficient being the Spectrum Light, with 400 to $800 \mathrm{~nm}, 0.18 \mathrm{~W} / \mathrm{cm}^{2}, 4$ minutes, 2 applications per day, for 12 weeks. The LED, with 625, 660, $850 \mathrm{~nm}, 2.4 \mathrm{~J} / \mathrm{cm} 2,50 \%, 5$ minutes, 3 applications per week, for 8 weeks. LLLT, with $685 \mathrm{~nm}$, $0.05 \mathrm{~W} / \mathrm{cm}^{2}, 10 \mathrm{~J} / \mathrm{cm} 2,3$ minutes, 6 applications per week, lasting from 2 to 20 weeks. And CAV, with $100 \mathrm{pps,}$ 0.24 or $0.25 \mathrm{~A}, 154 \mu \mathrm{s}, 100 \mathrm{~V}, 250$ or $360 \mu \mathrm{C} / \mathrm{s}, 50$ minutes, 5 applications per week, lasting 6 or 8 weeks.
} 
Keywords: Healing; Phototherapy; Pressure ulcer; Diabetic foot; Electrical stimulation therapy.

\section{Resumen}

Objetivo: Señalar los parámetros utilizados para la cicatrización de heridas mediante modalidades electrofototerapéuticas en pacientes con úlceras por presión y diabéticos. Métodos: Se realizaron búsquedas de artículos en las bases de datos PubMed, BVS, SciELO y PEDro, publicados entre 2010 y mayo de 2020. Dos autores analizaron de forma independiente los criterios de elegibilidad mediante la Escala PEDro. Resultados: Los estudios incluidos indican que los recursos electrofototerapéuticos pueden ser efectivos en el tratamiento de las úlceras por presión y diabéticas y que estos recursos aplicados junto con la intervención convencional optimizan la mejora del paciente. Conclusiones: Hubo una gran heterogeneidad en los parámetros analizados, siendo el más eficiente el Spectrum Light, con 400 a $800 \mathrm{~nm}, 0,18 \mathrm{~W} / \mathrm{cm}^{2}, 4$ minutos, 2 aplicaciones al día, durante 12 semanas. El LED, con $625,660,850 \mathrm{~nm}, 2,4 \mathrm{~J} / \mathrm{cm} 2,50 \%, 5$ minutos, 3 aplicaciones por semana, durante 8 semanas. LLLT, con $685 \mathrm{~nm}$, $0.05 \mathrm{~W} / \mathrm{cm}^{2}, 10 \mathrm{~J} / \mathrm{cm} 2,3$ minutos, 6 aplicaciones por semana, con una duración de 2 a 20 semanas. Y CAV, con $100 \mathrm{pps}, 0,24$ o $0,25 \mathrm{~A}, 154 \mu \mathrm{s}, 100 \mathrm{~V}, 250$ o $360 \mu \mathrm{C} / \mathrm{s}, 50$ minutos, 5 aplicaciones por semana, con una duración de 6 u 8 semanas.

Palabras clave: Curación; Fototerapia; Lesión por presión; Pie diabético; Terapia de estimulación eléctrica.

\section{Introdução}

A interrupção ou descontinuidade da pele pode caracterizar uma ferida, que é um problema bastante comum entre as pessoas, pois pode afetar qualquer indivíduo independentemente da idade, raça ou classe social (Evangelista et al., 2012; Leal \& Carvalho, 2012). Após um dano, o desenvolvimento do processo de reparação tecidual segue uma série de eventos bioquímicos e celulares que buscam restaurar o tecido, onde qualquer desequilíbrio, carência ou insuficiência de recursos, podem afetar a cicatrização (García-Pérez et al., 2018; Ruh et al., 2018; Souza et al., 2018; Vitoriano et al., 2019).

As lesões por pressão (LPPs - também denominadas úlceras por pressão, úlceras de decúbito, úlceras por compressão ou escaras) e as úlceras diabéticas (UDs), são condições multifatoriais resultantes do desequilíbrio metabólico da célula, que acarretam disfunções no processo de cicatrização, onde longos períodos de inflamação, dor, infecção, cronicidade, amputação e morte são complicações possíveis (García-Pérez et al., 2018; Vitoriano et al., 2019). Essas lesões podem afetar não só os tecidos subcutâneos, como também comprometer elementos musculares e ósseos (Polak et al., 2018; Rosa et al., 2019).

As lesões originárias do diabetes mellitus afetam com mais frequência os membros inferiores, sobretudo o pé (Rosa et al., 2019), e as lesões por pressão desenvolvem-se com maior frequência em calcanhares, tornozelos, quadris e no cóccix (Polak et al., 2018).

Os mecanismos de reparação tecidual podem ser influenciados pela fisioterapia, pois, a eletroterapia e a fototerapia (comumente denominadas em conjunto como eletrofototerapia) são modalidades terapêuticas que podem ser usados nos vários tipos de danos que podem vir a ocorrer aos tecidos biológicos, sendo que quando utilizados de forma adequada aceleram o processo de reparo tecidual (Souza et al., 2018; Silva et al., 2019), atuam no controle da dor, controle bacteriano e ainda podem produzir uma cicatrização esteticamente satisfatória (Moura et al., 2014).

A fototerapia técnica na qual utiliza-se a luz para realizar a biofotomodulação pode ser utilizada no tratamento de diferentes tipos de ferida, esta luz deve ser aplicada sobre a região a ser tratada com o comprimento de onda e a energia necessária para proporcionar a reparação do tecido, no caso das feridas utiliza-se na maioria das vezes espectro vermelho por ter maior interação com o tecido biológico (Silva et al., 2019). Dentre os recursos fototerapêuticos temos o laser de baixa intensidade (LLLT) (Santos et al., 2018), o LED (light emitting diode) (Rosa et al., 2019; Vitoriano et al., 2019). Já a eletroterapia, utiliza da aplicação de correntes elétricas no tratamento das feridas, sem geração de calor perceptível (Souza et al., 2018). Entre seus recursos temos a estimulação elétrica nervosa transcutânea (TENS) (García-Pérez et al., 2018), corrente contínua de baixa intensidade (CCBI) (Mohajeri-Tehrani \& Annabestani, 2014), e a corrente de alta voltagem (CAV) (Karsli et al., 2017).

Todos esses recursos atuam a nível celular, sendo indicados em vários casos referentes a danos teciduais (Landau et 
al., 2011; Leal \& Carvalho, 2012; Santos et al., 2018; Souza et al., 2018; Silva et al., 2019). Nesta revisão sintetizou-se evidências sob os parâmetros utilizados para a cicatrização de feridas através de modalidades eletrofototerapêuticas em pacientes com LPPs e UDs aplicando sob esses estudos a avaliação metodológica através da Escala PEDro.

\section{Metodologia}

Trata-se de uma revisão de escopo, elaborada de acordo com a metodologia do Joanna Briggs Institute (JBI) (Peters et al., 2020).

\subsection{Pergunta de revisão}

A pergunta norteadora desta revisão foi: Quais os melhores parâmetros das modalidades eletrofototerapêuticas utilizados para a cicatrização de feridas/úlceras diabéticas e por pressão?

\subsection{Critérios de elegibilidade}

\subsubsection{Participantes}

Considerou-se estudos envolvendo o tratamento eletrofototerapêutico para a cicatrização de feridas em pacientes com LPPs e UDs (independentemente de sua etiologia), de ambos os sexos e etnia, com idade acima de 18 anos.

\subsubsection{Conceito}

Considerou-se os recursos das modalidades de eletroterapia (ou eletroestimulação) utilizadas para cicatrização de feridas como TENS, CCBI, e CAV bem como os recursos da fototerapia, como a LLLT, LED e luz de espectro.

\subsubsection{Contexto}

Foram incluídos estudos desenvolvidos em todos os contextos em saúde como atenção básica, ambulatorial, hospitalar e cuidados intensivos, sem restrição de contexto geográfico e socioeconômico.

\subsection{Tipos de estudo}

Incluiu-se estudos experimentais como ensaios clínicos randomizados, ensaios clínicos controlados, ensaios clínicos pragmáticos e ensaios quase-experimentais ou do tipo antes de depois, que foram publicados em inglês, português e espanhol (por serem idiomas compreendidos pelos autores), com o período de publicação entre 2010 e maio de 2020, portanto, publicadas nos últimos 10 anos.

Estudos de revisão, observacionais, qualitativos, econômicos, cartas ao editor, opiniões de especialistas e resumos de conferências foram excluídos.

\subsection{Estratégia de busca}

As buscas foram realizadas na segunda quinzena de maio de 2020, através dos seguintes termos de busca registrados nos Descritores em Ciência da Saúde: "eletroterapia" OR "terapia de estimulação elétrica" OR "fototerapia" AND "ferida" OR "ferimentos e lesões" OR "lesão por pressão" OR "úlcera na pele" OR "úlcera diabética" OR "cicatrização", tanto em inglês, quanto em espanhol. Nos casos em que o texto completo estava indisponível, tentou-se o contato com os autores do estudo.

\subsection{Fontes de informação}

As buscas por estudos publicados foram realizadas nos seguintes portais e bases de dados: Biblioteca Virtual em 
Saúde (BVS), Pubmed, PEDro, SciELO.

\subsection{Seleção de estudos}

Pesquisas preliminares nas fontes de informações incluídas foram realizadas por dois revisores de forma independente, seguindo aos critérios de elegibilidade determinados para a revisão: ano de publicação, design do estudo, participantes, intervenções e desfechos de interesse. Os artigos que não apresentaram o título e o resumo elucidativos, foram analisados na íntegra, para que não houvesse a exclusão de estudos importantes para a revisão. Após esse passo, os artigos relevantes foram organizados e encaminhados para o gerenciador de referências Mendeley Desktop (Mendeley Ltd., Elsevier, Netherlands), e as duplicatas foram removidas. Em seguida, os artigos foram analisados rigorosamente, para verificar a elegibilidade de cada um, segundo o determinado para esta revisão.

O motivo da exclusão de estudos em texto completo que não atenderam aos critérios de elegibilidade foram registrados. Quaisquer divergências existentes entre os revisores no processo de seleção dos estudos foram sanadas mediante consenso, ou com um terceiro revisor. Foi elaborado um fluxograma baseado no PRISMA Extension for Scoping Reviews: Checklist and Explanation (PRISMA-ScR) (Tricco et al., 2018), para detalhar esse processo (Figura 1).

Figura 1. Fluxograma demonstrando o processo de busca e seleção dos estudos.

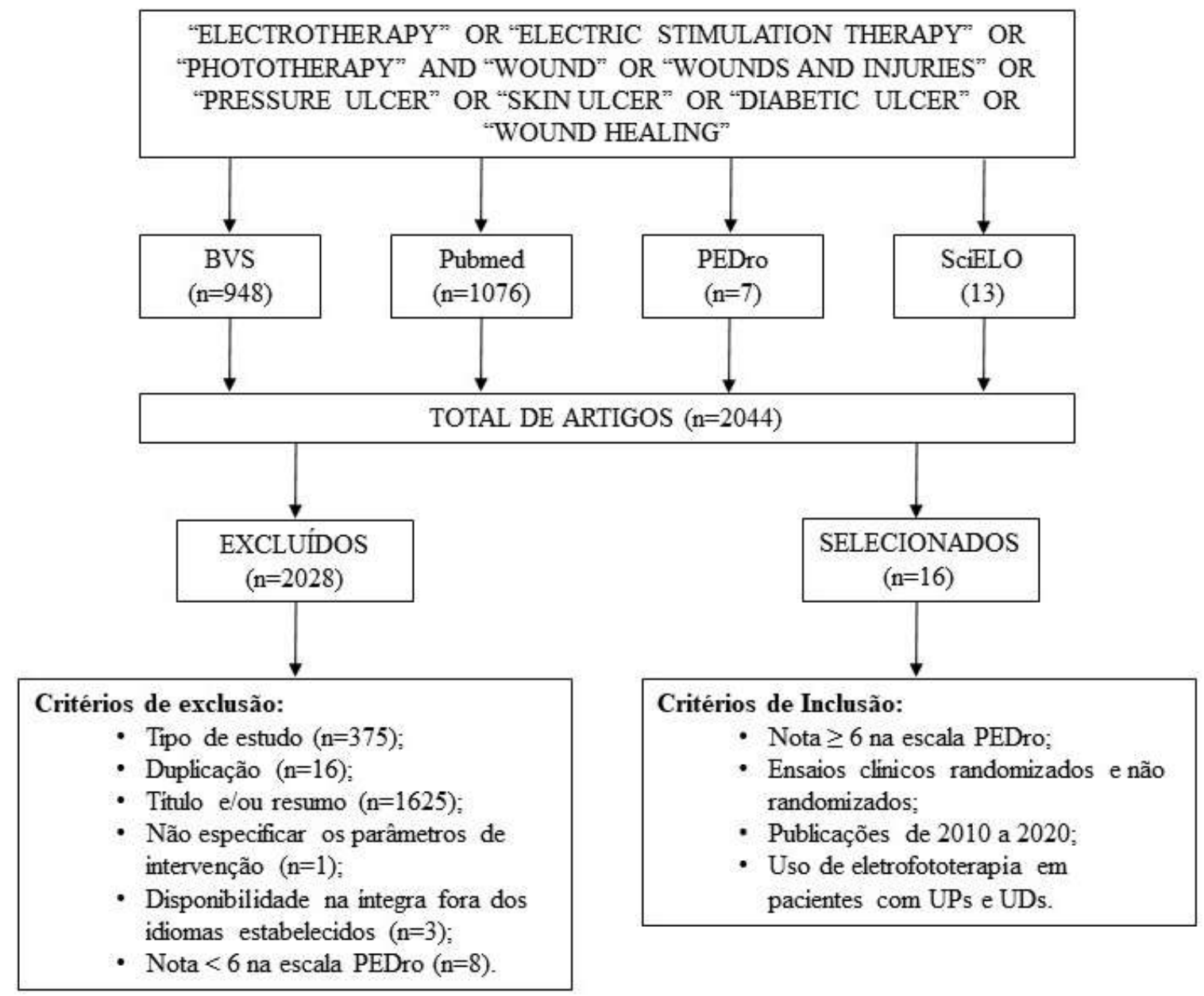

Fonte: Autores. 


\subsection{Avaliação da qualidade metodológica}

Utilizou-se a Escala PEDro, traduzida para o português, a qual possui critérios metodológicos que totalizam 10 pontos. Para cada indicador de qualidade na escala é atribuído um ponto (1) e zero ponto (0), o primeiro item não pontua, somente do item 2 ao 11, totalizando 11 itens (Shiwa et al., 2011).

Os artigos selecionados sem a nota da PEDRo foram avaliados por dois examinadores de forma independente quanto à qualidade metodológica. Sendo assim, permaneceram no estudo os artigos que apresentaram pontuação $\geq 6$, sendo que as discordâncias entre os revisores foram sanadas com a presença de um terceiro revisor.

\section{Resultados}

Foram incluídos 16 estudos para tabulação de dados, após os processos de seleção. Dentre esses, os protocolos terapêuticos com uso da fototerapia e eletroterapia para LPPs e UDs, utilizaram as modalidades LLLT, LED, luz de espectro, CAV, CCBI, e TENS combinados com o tratamento padrão de feridas (TPF). Os principais parâmetros e os mais eficazes, descritos na literatura estão na Tabela 1. 
Tabela 1 - Resumo das evidências das modalidades eletrofototerapêuticas para pacientes com LPPs e UDs.

\begin{tabular}{|c|c|c|c|c|c|c|c|}
\hline $\begin{array}{c}\text { Autor(es) } \\
\text { Ano }\end{array}$ & Objetivo & Amostra & Metodologia & $\begin{array}{l}\text { ogia } \\
\text { Intervenção }\end{array}$ & Resultados & Conclusão & $\begin{array}{l}\text { Escore } \\
\text { PEDro }\end{array}$ \\
\hline \multicolumn{8}{|c|}{$\begin{array}{l}\text { FOTOTERAPIA } \\
\end{array}$} \\
\hline $\begin{array}{l}\text { Landau at al. } \\
\text { (2011) }\end{array}$ & $\begin{array}{l}\text { Avaliar a eficácia da } \\
\text { fototerapia no tratamento de } \\
\text { úlceras nas pernas ou pés. }\end{array}$ & $\begin{array}{l}\mathrm{N}=16, \mathrm{AS}, \\
\text { média de } 62,9 \\
\text { anos, com } \\
\text { úlceras venosas } \\
\text { nas pernas ou } \\
\text { UDs nos pés. }\end{array}$ & $\begin{array}{l}\text { Ensaio Clínico } \\
\text { randomizado, duplo- } \\
\text { cego, placebo } \\
\text { controlado }\end{array}$ & $\begin{array}{l}\text { G1: Protótipo emissor de luz de } \\
\text { espectro ( } 400-800 \mathrm{~nm}, 0,18 \\
\text { W/cm², } 4 \mathrm{~min}, 2 / \text { dia, durante } 12 \\
\text { sem) + TPF; } \\
\text { G2: Placebo + TPF; } \\
\text { Foi avaliado a ASF de acordo com } \\
\text { a FDA (largura e comprimento). }\end{array}$ & $\begin{array}{l}\text { O G1 obteve uma maior } \\
\text { taxa de cura quando } \\
\text { comparado ao grupo } 2 \text {, } \\
89 \% \text { versus } 54 \% \text {, } \\
\text { respectivamente. Com } \\
\text { taxa de cura em } 90 \% \text { dos } \\
\text { pacientes do grupo } 1 \text {, } \\
\text { contra } 33 \% \text { do G2. }\end{array}$ & $\begin{array}{l}\text { O G1 } \\
\text { demonstrou uma } \\
\text { significativa } \\
\text { taxa de cura e } \\
\text { redução da área } \\
\text { da ferida. }\end{array}$ & $9 / 10$ \\
\hline $\begin{array}{l}\text { Frangež et al. } \\
(2018)\end{array}$ & $\begin{array}{l}\text { Determinar se a fototerapia } \\
\text { com LED melhora e reduz o } \\
\text { processo de cicatrização de } \\
\text { feridas crônicas em } \\
\text { pacientes diabéticos. }\end{array}$ & $\begin{array}{l}\mathrm{N}=60, \mathrm{AS}, \mathrm{com} \\
\text { UDs do joelho. }\end{array}$ & $\begin{array}{l}\text { Estudo prospectivo, } \\
\text { randomizado, duplo- } \\
\text { cego. }\end{array}$ & $\begin{array}{l}\text { G1: LED }(625,660,850 \mathrm{~nm}, 2,4 \\
\text { J/cm² }, 50 \%, 5 \text { min, } 3 / \text { sem, durante } \\
8 \text { sem })+ \text { TPF; } \\
\text { G2: Placebo + TPF; } \\
\text { Avaliação da ASF: Não informado. }\end{array}$ & $\begin{array}{l}\text { O G1 obteve redução da } \\
\text { superfície da ferida, mas } \\
\text { não foi } \\
\text { significativamente mais } \\
\text { rápida que o G2. }\end{array}$ & $\begin{array}{l}\text { O tratamento } \\
\text { com LED } \\
\text { oferece aos } \\
\text { pacientes } \\
\text { diabéticos uma } \\
\text { melhor chance } \\
\text { de recuperação } \\
\text { total. }\end{array}$ & $8 / 10$ \\
\hline $\begin{array}{l}\text { Kaviani et al. } \\
\text { (2011) }\end{array}$ & $\begin{array}{l}\text { Demonstrar que a LLLT } \\
\text { promove a cicatrização de } \\
\text { úlceras em pacientes com } \\
\text { diabetes mellitus. }\end{array}$ & $\begin{array}{l}\mathrm{N}=23, \mathrm{AS}, \text { com } \\
\text { UDs no pé. }\end{array}$ & $\begin{array}{l}\text { Ensaio clínico } \\
\text { controlado, duplo } \\
\text { cego. }\end{array}$ & $\begin{array}{l}\text { G1: LLLT }\left(685 \mathrm{~nm}, 0,05 \mathrm{~W} / \mathrm{cm}^{2},\right. \\
10 \mathrm{~J} / \mathrm{cm}^{2}, 3 \mathrm{~min} \text { e } 20 \mathrm{~s}, 6 / \mathrm{sem}, \\
\text { durante } 2 \text { à } 20 \mathrm{sem})+\mathrm{TPF} ; \\
\text { G2: Placebo + TPF; } \\
\text { Foi avaliado a ASF em } \mathrm{cm}^{2} \text { através } \\
\text { do software AutoCAD } 2000 .\end{array}$ & $\begin{array}{l}\text { Após } 4 \text { sem o G1 ( } 66,6 \% \\
\text { alcançaram cura) obteve } \\
\text { uma taxa de fechamento } \\
\text { das úlceras } \\
\text { significativamente maior } \\
\text { do que no G2 }(38,4 \% \\
\text { alcançaram cura). }\end{array}$ & $\begin{array}{l}\text { A LLLT pode } \\
\text { acelerar o } \\
\text { processo de } \\
\text { cicatrização das } \\
\text { úlcera não } \\
\text { isquêmicas de } \\
\text { pé diabético. }\end{array}$ & $8 / 10$ \\
\hline $\begin{array}{l}\text { Rosa et al. } \\
(2019)\end{array}$ & $\begin{array}{l}\text { Analisar os efeitos do } \\
\text { sistema Rapha (SR) na } \\
\text { cicatrização de úlceras } \\
\text { neuropáticas associadas ao } \\
\text { pé diabético. }\end{array}$ & $\begin{array}{l}\mathrm{N}=15, \mathrm{AS}, 30- \\
59 \text { anos, com } \\
\text { UDs no pé. }\end{array}$ & $\begin{array}{l}\text { Ensaio clínico } \\
\text { randomizado, } \\
\text { controlado e cego. }\end{array}$ & $\begin{array}{l}\text { G1: SR ( } 635-640 \mathrm{~nm}, 1,8 \mathrm{~W} / \mathrm{cm}^{2} \text {, } \\
25 \mathrm{~J} / \mathrm{cm}^{2}, 35 \mathrm{~min} \text {, durante } 6 \mathrm{sem} . \mathrm{A} \\
\text { biomembrana de látex natural foi } \\
\text { aplicado pelo profissional ou pelo } \\
\text { paciente sobra a úlcera + TPF; } \\
\text { G2: Controle (TPF); } \\
\text { Á ASF foi avaliada via fotografia } \\
\text { digitais. }\end{array}$ & $\begin{array}{l}\text { O G1 obtive uma maior } \\
\text { taxa de granulação do } \\
\text { tecido e maior eficácia } \\
\text { no reparo tecidual das } \\
\text { lesões quando } \\
\text { comparados ao G2. }\end{array}$ & $\begin{array}{l}\text { O SR provou ser } \\
\text { eficaz, mesmo } \\
\text { quando } \\
\text { autoaplicado. }\end{array}$ & $7 / 10$ \\
\hline
\end{tabular}


Mathur et al. Avaliar a eficácia da LLLT N=30, AS, 49- Ensaio clínico (2017) para o tratamento de úlceras 54 anos, UDs no randomizado do pé diabético em um pé. centro de atendimento terciário.
G1: LLLT $\left(660 \mathrm{~nm}, 0,05 \mathrm{~W} / \mathrm{cm}^{2}, 3\right.$ J, 5 a 8 pontos, 60s/ponto, mínimo de 5 min, durante 15 dias) + TPF;

G2: Controle (TPF);

A ASF foi análise por fotografias digitais.

A ASF foi avaliada através do software Image $\mathrm{J} 囚$.

(2014)

Comparar os efeitos da TPF na cicatrização de úlceras nos pés diabéticos. anos, com UDs

no pé.

Ensaio clínico

controlado randomizado.
G2: CAV pulsada $(100 \mu \mathrm{s}, 100$ pps, 45 min, durante 16 sem) +

\begin{tabular}{|c|c|c|c|c|}
\hline $\begin{array}{l}\text { Feitosa et al. } \\
(2015)\end{array}$ & $\begin{array}{l}\text { Avaliar os efeitos da LLLT } \\
\text { aplicando no reparo tecidual } \\
\text { em portadores de úlcera } \\
\text { devido a diabetes. }\end{array}$ & $\begin{array}{l}\mathrm{N}=16, \mathrm{AS}, 60- \\
95 \text { anos, com } \\
\text { UDs no pé. }\end{array}$ & $\begin{array}{l}\text { Estudo clínico, } \\
\text { controlado, } \\
\text { randomizado, } \\
\text { intervencionista, } \\
\text { quali-quantitativo. }\end{array}$ & $\begin{array}{l}\text { G1: LLLT }\left(632,8 \mathrm{~nm}, 4 \mathrm{~J} / \mathrm{cm}^{2} \text {, }\right. \\
\text { 80s/ponto, } 0,03 \mathrm{~W} / \mathrm{cm}^{2}, 3 / \mathrm{sem} \text {, } \\
\text { durante } 3 \mathrm{sem})+\mathrm{TPF} ; \\
\text { G2: Controle (TPF); }\end{array}$ \\
\hline
\end{tabular}

Ortíz et al. Comparar os efeitos da $\quad 28$ P, 30-75 Ensaio clínico $\quad$ G1: Controle (TPF)
LLLT, e a CAV pulsada e
G1: Obteve aumento na quantidade de granulação no tecido lesado e FPF;

G2: Ineficaz.

\section{G1: Diminuição do edema e FPF;}

G2. Aumento no tamanho das feridas. benéfica como TPF;

G3: LLLT (685 nm, 1,5 a $2 \mathrm{~J} / \mathrm{cm}^{2}$, $0,03 \mathrm{~W} / \mathrm{cm}^{2}$, durante $\left.16 \mathrm{sem}\right)+$ TPF;

A avaliação da ASF com papel milimetrado e digitalização usando Image 1,39 usoftware (National Institutes of Health, USA).

$\begin{array}{ll}\text { Chaves et al. } & \text { Verificar se a termografia } \\ \text { pode ser utilizada como } \\ \text { método de avaliação da } \\ \text { cicatrização de LPPs. }\end{array}$
$\begin{array}{ll}\mathrm{N}=8,40-80 & \text { Ensaio clínico } \\ \text { anos, com LPPs. } & \text { controlado. }\end{array}$ cicatrização de LPPs.
Os resultados sugerem que a LLLT é adjuvante ao

TPF no

tratamento de

UDs no pé..

O LLLT parece

indolor e de

baixo custo,

sobre o reparo das UDs no pé.

Os resultados deste estudo não

$\begin{array}{lll}\text { A proporção de cura na } & \text { Os resultados } \\ \text { semana } 4,8,12 \text { e } 16 & \text { deste estudo não } \\ \text { aumentaram em todos os } & \text { demonstram } \\ \text { grupos. Não houve } & \text { efeitos } \\ \text { diferenças significativas } & \text { adicionais do } \\ \text { entre os grupos. } & \text { LLLT ou da } \\ & \text { CAV pulsada na } \\ & \text { recuperação das } \\ & \text { UDs. }\end{array}$

G1: Aumento na área e profundidade da úlcera;

A termografia pode ser considerada um G2: Fechamento parcial da ferida e melhora na irrigação local. método adjuvante na avaliação do processo de cicatrização de feridas.

\section{G1: Controle (TPF);}

G2: LED (660-940 nm, $4 \mathrm{~J} / \mathrm{cm}^{2}$, $50 \%, 5 \mathrm{~min}, 3 / \mathrm{sem}$, durante $4 \mathrm{sem}$ ) $+\mathrm{TPF}$

A avaliação da ASF ocorreu com câmera Nikon® D5100 e termogramas da ferida (Câmera termográfica FLIR I60®). 


\begin{tabular}{|c|c|c|c|c|c|c|c|}
\hline \multirow[b]{2}{*}{$\begin{array}{l}\text { Polak et al. } \\
\text { (2018) }\end{array}$} & \\
\hline & $\begin{array}{l}\text { Avaliar os efeitos da CAV } \\
\text { pulsada monofásica } \\
\text { catódica e anodal sobre a } \\
\text { ferida. }\end{array}$ & $\begin{array}{l}\mathrm{N}=61, \mathrm{AS},>18 \\
\text { anos, com LPPs } \\
\text { em pessoas com } \\
\text { lesões } \\
\text { neurológicas. }\end{array}$ & $\begin{array}{l}\text { Estudo clínico } \\
\text { randomizado, cego, } \\
\text { controlado. }\end{array}$ & $\begin{array}{l}\text { G1: CAV catódica + TPF; } \\
\text { G2: CAV anódica + TPF; } \\
\text { G3: Placebo (TPF); } \\
\text { CAV: } 0,25 \mathrm{~A}, 100 \mathrm{pps}, 154 \mu \mathrm{s}, 100 \\
\mathrm{~V}, 360 \mu \mathrm{C} / \mathrm{s}, 50 \mathrm{~min} / \mathrm{dia}, 5 / \mathrm{sem}, \\
\text { durante } 8 \mathrm{sem} \text {; } \\
\text { Foi avaliada ASF através de um } \\
\text { digitalizador, sendo que o fluxo } \\
\text { sanguíneo foi avaliado através de } \\
\text { fluxometria Doppler a laser. }\end{array}$ & $\begin{array}{l}\text { G1 e G2: Fechamento } \\
\text { parcial da ferida; } \\
\text { G3: Ineficaz. }\end{array}$ & $\begin{array}{l}\text { A CAV anodal e } \\
\text { catodal, } \\
\text { promovem a } \\
\text { cura das úlceras } \\
\text { em pacientes } \\
\text { com lesões } \\
\text { neurológicas. } \\
\text { No entanto, não } \\
\text { afetaram o fluxo } \\
\text { sanguíneo da } \\
\text { ferida. }\end{array}$ & $9 / 10$ \\
\hline $\begin{array}{l}\text { Polak et al. } \\
\text { (2017) }\end{array}$ & $\begin{array}{l}\text { Comparar a eficácia da } \\
\text { estimulação elétrica } \\
\text { catódica versus catódica }+ \\
\text { anodal no tratamento de } \\
\text { LPPs. }\end{array}$ & $\begin{array}{l}\mathrm{N}=63, \mathrm{AS}, 60- \\
95 \text { anos, com } \\
\text { LPPs na cintura } \\
\text { pélvica. }\end{array}$ & $\begin{array}{l}\text { Estudo clínico } \\
\text { randomizado } \\
\text { controlado. }\end{array}$ & $\begin{array}{l}\text { G1: CAV catódica + TPF; } \\
\text { G2: CAV catódica + anódica + } \\
\text { TPF; } \\
\text { G3: Placebo (TPF); } \\
\text { CAV: } 0,25 \mathrm{~A}, 100 \mathrm{pps}, 154 \mu \mathrm{s}, 100 \\
\mathrm{~V}, 250 \mu \mathrm{C} / \mathrm{s}, 50 \mathrm{~min}, 5 / \mathrm{sem}, \\
\text { durante } 6 \mathrm{sem} \text {; } \\
\text { Foi avaliado a ASF pelo } \\
\text { Digitalizador Mutoh Kurta XGT, } \\
\text { Altek, EUA. }\end{array}$ & $\begin{array}{l}\text { G1 e G2: Redução da } \\
\text { ASF, acelerando sua } \\
\text { cura. } \\
\text { G3: Ineficaz. }\end{array}$ & $\begin{array}{l}\text { A CAV com } \\
\text { cátodo como } \\
\text { único eletrodo } \\
\text { de tratamento } \\
\text { ou o cátodo na } \\
\text { primeira semana } \\
\text { e depois o } \\
\text { ânodo são } \\
\text { eficazes no } \\
\text { tratamento de } \\
\text { LPPs. }\end{array}$ & $9 / 10$ \\
\hline $\begin{array}{l}\text { Polak et al. } \\
\text { (2016) }\end{array}$ & $\begin{array}{l}\text { Investigar a eficácia da } \\
\text { CAV pulsada monofásica } \\
\text { como complemento de um } \\
\text { TPF das LPPs nos estágios } \\
\text { II e III. }\end{array}$ & $\begin{array}{l}\mathrm{N}=49, \text { AS, } 60- \\
95 \text { anos, com } \\
\text { LPPs. }\end{array}$ & $\begin{array}{l}\text { Ensaio clínico } \\
\text { randomizado } \\
\text { controlado, duplo- } \\
\text { cego. }\end{array}$ & $\begin{array}{l}\text { G1: CAV pulsada monofásica (100 } \\
\text { V, } 154 \mu \mathrm{s} ; 100 \mathrm{pps}, 0,24 \mathrm{~A}, 250 \\
\mu / \mathrm{C}, 50 \mathrm{~min} / \text { dia, } 5 / \mathrm{sem} \text {, durante } 6 \\
\text { sem) + TPF; } \\
\text { G2: Placebo (TPF); } \\
\text { A gravidade da ferida foi } \\
\text { estabelecida com base nos critérios } \\
\text { do Painel Consultivo Nacional } \\
\text { sobre LPPs. }\end{array}$ & $\begin{array}{l}\text { O G1 obteve redução } \\
\text { percentual da área da } \\
\text { ferida em } 6 \text { semanas de } \\
\text { intervenção, com } \\
\text { aumento significativo da } \\
\text { taxa de cicatrização de } \\
\text { feridas quando } \\
\text { comparado ao G2. }\end{array}$ & $\begin{array}{l}\text { O estudo } \\
\text { mostrou que a } \\
\text { CAV pulsada } \\
\text { monofásica é } \\
\text { eficaz no } \\
\text { tratamento das } \\
\text { LPPs, reduzindo } \\
\text { sua área e } \\
\text { acelerando sua } \\
\text { cicatrização. }\end{array}$ & $9 / 10$ \\
\hline
\end{tabular}


Polak et al.

(2016)

$\begin{array}{llll}\begin{array}{l}\text { Polak et al. } \\ \text { (2016) }\end{array} & \begin{array}{l}\text { Determinar se usando o } \\ \text { UST e CAV pulsada }\end{array} & \begin{array}{l}\mathrm{N}=77, \mathrm{AS}, 60- \\ 95 \text { anos, com } \\ \text { monofásica, a taxa de }\end{array} & \begin{array}{l}\text { Estudo clínico } \\ \text { randomizado } \\ \text { mudança na área das LPPs } \\ \text { controlado, cego. }\end{array} \\ \begin{array}{l}\text { te pacientes mais velhos } \\ \text { pode ser acelerada. }\end{array} & \begin{array}{l}\text { membro } \\ \text { inferior. }\end{array} & \\ & \end{array}$

$\begin{array}{ll}\mathrm{N}=67, \mathrm{AS}, & \text { Estudo prospectivo, } \\ \text { média de } 50 & \text { randomizado, cego, } \\ \text { anos, com LPPs. } & \text { clínico controlado. }\end{array}$

anos, com LPPs. clínico controlado.

administrada como parte de um programa

terdisciplinar no

pessoas com lesão medular.
G1: CAV pulsada monofásica (100 G1 e G2: Redução da pps, $154 \mu \mathrm{s}, 100 \mathrm{~V}, 250 \mu \mathrm{C} / \mathrm{s}, 50$

min, $1 /$ dia, $5 /$ sem, durante 6 sem) + TPF;

G2: UST (1MHz, 0,5 W/cm ${ }^{2}, 1-3$ $\mathrm{min} / \mathrm{cm}^{2}, 1 / \mathrm{dia}, 5 / \mathrm{sem}$, durante 6 sem) + TPF;

\section{G3: TPF;}

A ASF foi avaliada pelo

digitalizador Mutoh Kurta XGT,

Altek, EUA).

G1: CAV pulsada $(50 \mu \mathrm{s}, 50 \mathrm{e}$

$150 \mathrm{~V}, 20 \mathrm{~min}, 100 \mathrm{~Hz}$, seguida de

$20 \mathrm{~min}, 10 \mathrm{~Hz}$ e $20 \mathrm{~min}$ fora do

ciclo a cada hora, $8 \mathrm{~h} /$ dia, durante

12 sem) + TPF;

G2: TPF;

Foram avaliadas alterações na aparência da ferida (fotográficas

Canon Rebel 300D EOS) incluindo uma régua autoadesiva com escala milimétrica e ferramenta Bates-

Jensen.

$25 \mathrm{~mA}, 60 \mathrm{~min}, 3 / \mathrm{sem}$, durante 2

ca e dor secundária

pressão crônica.
G1: TENS $(50 \mu \mathrm{s}, 40 \mathrm{~Hz}, 15 \mathrm{e}$ meses) + TPF

G2: Controle (TPF);

$\begin{array}{ll}\mathrm{N}=22, \mathrm{AS}, \text { com } & \begin{array}{l}\text { Estudo clínico } \\ \text { piloto. }\end{array} \\ \text { LPPs em } & \\ \text { membros } & \\ \text { inferiores. } & \end{array}$

inferiores.

A área de cada lesão foi medida com fita métrica.

\section{ASF.}

G3: Ineficaz.

O UST e CAV

pulsada

monofásica são

comparativamen

te mais eficazes

na redução da

área superficial

das LPPs em

pessoas idosas,

do que o TPF

sozinho.

$\begin{array}{ll}\begin{array}{l}\text { O G2 obteve redução } \\ \text { percentual }\end{array} & \begin{array}{l}\text { A aplicação da } \\ \text { terapia por CAV } \\ \text { significativamente maior } \\ \text { pulsada, } \\ \text { tratamento comparado ao }\end{array} \\ \begin{array}{l}\text { G2, sendo que neste } \\ \text { pode ser } \\ \text { integrado com } \\ \text { sucesso em um } \\ \text { tamanho de 4 feridas. }\end{array} & \begin{array}{l}\text { programa de } \\ \text { tratamento de } \\ \text { feridas, pois } \\ \text { acelera a } \\ \text { redução do } \\ \text { tamanho da } \\ \text { ferida. }\end{array} \\ \text { G1: Diminuição } & \text { Os resultados } \\ \text { mostraram } \\ \text { significativa da dor, } & \text { melhora no } \\ \text { temperatura da pele e } & \text { índice da taxa } \\ \text { aceleração da } & \text { de cura, os } \\ \text { cicatrização. } 80 \% \text { foram } & \text { níveis de dor e a } \\ \text { completamente curados } & \text { temperatura da } \\ \text { de acordo com o índice } & \text { pele } \\ \text { RESVECH. } & \text { melhoraram } \\ \text { G2: Ineficaz } & \text { significativamen } \\ \text { te no G1. }\end{array}$




\begin{tabular}{|c|c|c|c|c|c|c|c|}
\hline $\begin{array}{l}\text { Mohajeri- } \\
\text { Tehrani \& } \\
\text { Annabestani. } \\
\text { (2014) }\end{array}$ & $\begin{array}{l}\text { Investigou o efeito da CCBI } \\
\text { catódica na liberação do } \\
\text { fator de crescimento } \\
\text { endotelial vascular } \\
\text { plasmático e óxido nítrico } \\
\text { na ulceração do pé } \\
\text { diabético. }\end{array}$ & $\begin{array}{l}\mathrm{N}=33, \mathrm{AS}, 40- \\
60 \text { anos, com } \\
\text { UDs no } \\
\text { tornozelo e pé. }\end{array}$ & $\begin{array}{l}\text { Ensaio clínico } \\
\text { randomizado. }\end{array}$ & $\begin{array}{l}\text { G1: CCBI (1,48 mA, } 60 \text { min, } \\
\text { 3/sem., durante } 4 \text { sem) + TPF; } \\
\text { G2: Placebo (TPF); } \\
\text { Foram avaliadas a temperatura da } \\
\text { pele (Multímetro digital HiTester, } \\
\text { modelo 3801, Hioki EE Corp, } \\
\text { Nagano, Japão), e a área de } \\
\text { superfície da ferida (Régua métrica } \\
\text { e uma câmera digital). }\end{array}$ & $\begin{array}{l}\text { G1: Melhora na } \\
\text { temperatura da pele, } \\
\text { aumento na expressão do } \\
\text { fator de crescimento } \\
\text { endotelial vascular } \\
\text { plasmático e óxido } \\
\text { nítrico, proporcionando } \\
\text { fechamento parcial da } \\
\text { ferida; } \\
\text { G2: Ineficaz. }\end{array}$ & $\begin{array}{l}\text { A aplicação da } \\
\text { CCBI catódica } \\
\text { na intensidade } \\
\text { do limiar } \\
\text { sensorial, pode } \\
\text { induzir melhora } \\
\text { na temperatura } \\
\text { da pele e a } \\
\text { cicatrização da } \\
\text { feridas. }\end{array}$ & $6 / 10$ \\
\hline $\begin{array}{l}\text { Franek et al. } \\
(2012)\end{array}$ & $\begin{array}{l}\text { Avaliar o efeito de CAV em } \\
\text { LPPs não cicatrizantes, de } \\
\text { extremidade inferior, } \\
\text { estágio II e estágio III. }\end{array}$ & $\begin{array}{l}\mathrm{N}=50, \mathrm{AS}, 14- \\
88 \text { anos, com } \\
\text { LPPs nas } \\
\text { pernas, } \\
\text { tornozelos e pés. }\end{array}$ & $\begin{array}{l}\text { Ensaio clínico } \\
\text { randomizado e } \\
\text { controlado. }\end{array}$ & $\begin{array}{l}\text { G1. CAV }(100 \mathrm{~V}, 100 \mu \mathrm{s}, 100 \mathrm{pps} \text {, } \\
50 \mathrm{~min}, 1 / \text { dia, durante } 6 / \mathrm{sem})+ \\
\text { TPF; } \\
\text { G2. TPF; } \\
\text { Foram avaliados a profundidade, } \\
\text { área e o volume das úlceras. }\end{array}$ & $\begin{array}{l}\text { G1: Após } 6 \text { semanas de } \\
\text { tratamento foi observada } \\
\text { uma diminuição } \\
\text { estatisticamente } \\
\text { significativa no tamanho } \\
\text { da úlcera. } \\
\text { G2: Diminuição da área } \\
\text { da ferida. }\end{array}$ & $\begin{array}{l}\text { Os resultados } \\
\text { exibem } \\
\text { reduções } \\
\text { significativamen } \\
\text { te maiores na } \\
\text { área e volume } \\
\text { da superfície da } \\
\text { ferida submetida } \\
\text { a CAV. }\end{array}$ & $6 / 10$ \\
\hline
\end{tabular}

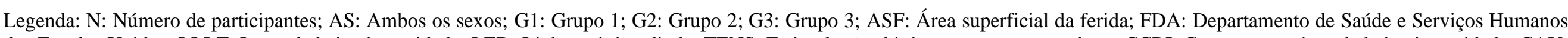

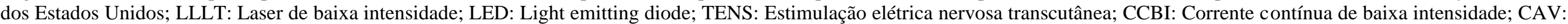

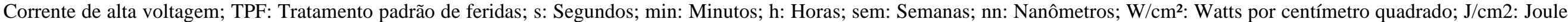

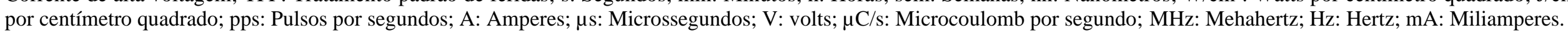
Fonte: Autores. 


\section{Discussão}

Há diferentes protocolos para a aplicação da eletrofototerapia em pacientes com LPPs e UDs. Todos os protocolos trabalharam de forma combinada com o TPF, associado com a limpeza da ferida (por exemplo: solução salina ou permanganato de potássio), desbridamento de tecido necrótico, curativos tópicos locais (com hidrogel, alginato, espuma de prata, colagenase/cloranfenicol, etc.) e medicamentos para controle da infecção e inflamação (Houghton et al., 2010; Kaviani et al., 2011; Landau et al., 2011; Franek et al., 2012; Mohajeri-Tehrani \& Annabestani, 2014; Ortíz et al., 2014; Chaves et al., 2015; Feitosa et al., 2015; Polak et al., 2016a, 2016b; Mathur et al., 2017; Polak et al., 2017; Frangež; Nizič-Kos \& Frangež, 2018; García-Pérez et al., 2018; Polak et al., 2018; Rosa et al., 2019).

Quanto aos parâmetros, ao se analisar os trabalhos pode-se perceber que os recursos mais utilizados na modalidade de eletrofototerapia foram a CAV (Houghton et al., 2010; Franek et al., 2012; Ortíz et al., 2014; Polak et al., 2016a, 2016b; Polak et al., 2017; Polak et al., 2018), seguido pelo LLLT (Kaviani et al., 2011; Ortíz et al., 2014; Feitosa et al., 2015; Mathur et al., 2017) e LED (Mohajeri-Tehrani \& Annabestani, 2014; Chaves et al., 2015; Rosa et al., 2019). Os parâmetros utilizados (mínimos e máximos, de acordo com os estudos) na CAV para a cicatrização de feridas foram: frequência de pulso de 100 pps, pico de corrente elétrica de 0,24 à $0,25 \mathrm{~A}$, duração de pulso 100 à $154 \mu \mathrm{s}$, voltagem de 50 à $150 \mathrm{~V}$, carga entregue pelos eletrodos de 250 à $360 \mu \mathrm{C} / \mathrm{s}$, tempo por intervenção de 20 à 50 minutos, 5 aplicações por semana, 6 à 12 semanas de duração total (Houghton et al., 2010; Franek et al., 2012; Ortíz et al., 2014; Polak et al., 2016a, 2016b; Polak et al., 2017; Polak et al., 2018). No LLLT foram: comprimento de onda entre 632,8 à $685 \mathrm{~nm}$, densidade de potência entre 0,03 à $0,05 \mathrm{~W} / \mathrm{cm}^{2}$, densidade de energia de 1,5 à $10 \mathrm{~J} / \mathrm{cm} 2$, com tempo de aplicação 5 minutos, sendo de 60 à 80 segundos/ponto, 3 à 6 aplicações por semana e duração total de 15 dias à 20 semanas (Kaviani et al., 2011; Ortíz et al., 2014; Feitosa et al., 2015; Mathur et al., 2017). No LED foram: comprimento de onda de 625 à $940 \mathrm{~nm}$, densidade de potência de 1,8 W/cm2, densidade de energia de 2 à $4 \mathrm{~J} / \mathrm{cm} 2$, modo pulsado de 50\%, 5 à 35 minutos, 3 à 12 aplicações semanais, durante 4 à 8 semanas (Mohajeri-Tehrani \& Annabestani, 2014; Chaves et al., 2015; Rosa et al., 2019).

Em sua maioria, os recursos das modalidades de fototerapia e eletroterapia, apresentam efeitos positivos no alívio da dor, diminuição do edema, redução significativa no comprimento e largura da superfície da ferida, melhoria dos marcadores bioquímicos envolvidos, melhoria na modulação da neovascularização e aceleração da proliferação, promovendo sua cura contínua (Houghton et al., 2010; Franek et al., 2012; Kaviani et al., 2011; Chaves et al., 2015; Feitosa et al., 2015; Polak et al., 2016a, 2016b; Mathur et al., 2017; Polak et al., 2017; Frangež; Nizič-Kos \& Frangež, 2018; Polak et al., 2018; Rosa et al., 2019).

Baseado na escala PEDro, os estudos com melhor qualidade metodológica ( $\geq 8$ ) foram: Landau et al (2011), no qual evidenciou-se que um protótipo emissor de luz de espectro (400 à $800 \mathrm{~nm}$ ), com densidade de potência de $0,18 \mathrm{~W} / \mathrm{cm}^{2}$, tempo de aplicação de 4 minutos, 2 aplicações por dia, durante 12 semanas, é capaz de aumentar significativamente a taxa de cura e reduzir a área de úlceras venosas e UDs.

Frangez et al (2018), utilizaram o LED com três diferentes comprimentos de onda (625, 660, 850 nm), densidade de energia de 2,4 J/cm2, modo pulsado de $50 \%$, por 5 minutos, 3 aplicações por semana, durante 8 semanas, e observaram melhorias na microcirculação, irrigação local e diminuição da dor, acelerando o processo de cura em pacientes com UDs.

Kaviani et al (2011), verificaram a eficácia do LLLT, com comprimento de onda de $685 \mathrm{~nm}$, densidade de potência $0,05 \mathrm{~W} / \mathrm{cm}^{2}$, densidade de energia de $10 \mathrm{~J} / \mathrm{cm} 2$, com tempo de aplicação 3 minutos, 6 aplicações por semana, com duração de 2 à 20, e observaram aceleração da cicatrização das úlceras não isquêmicas em pacientes com UDs.

Polak et al (2016a), Polak et al (2017), e Polak et al (2018), empregaram a CAV com frequência de pulso de 100 pps, pico de corrente elétrica de 0,24 ou 0,25 A, duração de pulso de $154 \mu$ s, voltagem de $100 \mathrm{~V}$, carga entregue pelos eletrodos de 
250 ou $360 \mu \mathrm{C} / \mathrm{s}$, tempo por intervenção de 50 minutos, 5 aplicações por semana, com duração de 6 ou 8 semanas e observaram uma redução significativa no comprimento e largura da superfície da ferida, promovendo sua cura contínua em pacientes com LPPs.

A maioria dos estudos inclusos nesta revisão apontam a eficácia dos recursos eletrofototerapêuticos no processo de cicatrização tecidual e limiar de dor. Pressupõe-se que estes recursos podem proporcionar significativas melhoras na redução da área da ferida, especialmente em comparação com os grupos submetidos ao tratamento usual/placebo.

Salienta-se que, as modalidades eletrofototerapêuticas devem ser empregadas como uma abordagem combinada, sempre sendo associadas ao tratamento usual de feridas, e não como a única abordagem terapêutica. Em sua maioria, estudos que realizaram intervenções mistas, isto é, eletrofototerapia associada ao tratamento usual, apresentaram resultados mais satisfatórios que o tratamento usual isolado.

\section{Conclusão}

A eletrofototerapia pode ser empregada para a cicatrização de feridas em pacientes com úlceras diabéticas e por pressão, promovendo redução no comprimento, largura e profundidade da ferida, melhora a vascularização, diminui edema, aumenta a expressão do fator de crescimento endotelial, melhora da temperatura da pele, melhora dos marcadores bioquímicos envolvidos e melhora da proliferação celular principalmente se forem usados os recursos como o protótipo de Luz de espectro, o LLLT, o LED e a CAV.

No que diz respeito aos parâmetros, verificou-se que existe uma grande heterogeneidade na sua dosimetria entre os protocolos analisados, sendo os mais eficazes: Luz de espectro com 400 à $800 \mathrm{~nm}, 0,18 \mathrm{~W} / \mathrm{cm}^{2}, 4$ minutos, 2 aplicações por dia, durante 12 semanas. O LED com 625, 660, $850 \mathrm{~nm}, 2,4 \mathrm{~J} / \mathrm{cm} 2,50 \%, 5$ minutos, 3 aplicações por semana, durante 8 semanas. A LLLT, com $685 \mathrm{~nm}, 0,05 \mathrm{~W} / \mathrm{cm}^{2}, 10 \mathrm{~J} / \mathrm{cm} 2,3$ minutos, 6 aplicações por semana, com duração de 2 à 20 semanas. E a CAV com 100 pps, 0,24 ou 0,25 A, $154 \mu \mathrm{s}, 100 \mathrm{~V}, 250$ ou $360 \mu \mathrm{C} / \mathrm{s}, 50$ minutos, 5 aplicações por semana, com duração de 6 ou 8 semanas.

\section{Implicações dos resultados para a prática clínica}

As evidências encontradas apontam que os melhores resultados se deram em protocolos que abordaram as modalidades eletrofototerapêuticas de forma combinada com o tratamento usual.

\section{Implicações dos resultados da pesquisa}

Recomenda-se que em estudos futuros haja uma melhor condução dos métodos de cegamento, alocação e homogeneização da amostra, com o intuito de desenvolver ensaios clínicos com boa qualidade metodológica.

\section{Agradecimentos}

Os autores deste estudo agradecem à Fundação de Amparo à Pesquisa do Amazonas (FAPEAM) e à Universidade Federal do Amazonas (UFAM) que através do Programa Institucional de Bolsas de Iniciação Científica (PIBIC) proporcionaram o desenvolvimento desta revisão. 


\section{Referências}

Chaves, M. E. A., Silva, F. S., Soares, V. P. C., Ferreira, R. A. M., Gomes, F. S. L., Andrade, R. M., \& Pinotti, M. (2015). Evaluation of healing of pressure ulcers through thermography: a preliminary study. Research on Biomedical Engineering, 31(1):3-9.

Evangelista, D. G., Magalhães, E. R. M., Moretão, D. I. C., Stival, M. M., \& Lima, L. R. (2012). Impacto das feridas crônicas na qualidade de vida de usuários da estratégia de saúde da família. Revista de Enfermagem do Centro Oeste Mineiro, 254-263.

Farcic, T. S., Lima, R. M. C. B., Machado, A. F. P., Baldan, C. S., Villicev, C. M., Junior, I. E., \& Masson, I. F. B. (2012). Aplicação do ultrassom terapêutico no reparo tecidual do sistema musculoesquelético. Arquivo Brasileira Ciências da Saúde, 37(3), 149-153.

Feitosa, M. C. P., Carvalho, A. F. M., Feitosa, V. C., Coelho, I. M., Oliveira, R. A., \& Arisawa, E. Â. L. (2015). Effects of the Low-Level Laser Therapy (LLLT) in the process of healing diabetic foot ulcers. Acta Cirúrgica Brasileira, 30(12):852-7.

Franek, A., Kostur, R., Polak, A., Taradaj, J., Szlachta, Z., Blaszczak, E., Dolibog, P., Dolibog, P., Koczy, B., \& Kucio, C. (2012). Using high-voltage electrical stimulation in the treatment of recalcitrant pressure ulcers: results of a randomized, controlled clinical study. Ostomy Wound Management, 58(3):3044.

Frangež, I., Nizič-Kos, T., \& Frangež, H. B. (2018). Phototherapy with LED Shows Promising Results in Healing Chronic Wounds in Diabetes Mellitus Patients: A Prospective Randomized Double-Blind Study. Photomedicine Laser Surgery, 36(7):377-82.

García-Pérez, S., García-Ríos, M. C., Pérez-Mármol, J. M., Tapia-Haro, R. M., Albornoz-Cabello, M., Valenza, M. C. \& Aguilar-Ferrándiz, M. E. (2018). Effectiveness of Transcutaneous Electrical Nerve Stimulation Energy in Older Adults: A Pilot Clinical Trial. Advances in Skin \& Wound Care, 31(10), 462-9.

Houghton, P. E., Campbell, K. E., Fraser, C. H., Harris, C., Keast, D. H., Potter, P. J., Hayes, K. C., \& Woodbury, M. G. (2010). Electrical stimulation therapy increases rate of healing of pressure ulcers in community-dwelling people with spinal cord injury. Archives of Physical Medicine and Rehabilitation, 91(5):669-78

Karsli, P. B., Gurcay, E., Karaahmet, O. Z., \& Cakci, A. (2017). High-Voltage electrical stimulation versus ultrasound in the treatment of pressure ulcers. Advances in Skin \& Wound Care, 30(12), 565-70.

Kaviani, A., Djavid, G. E., Ataie-Fashtami, L., Fateh, M., Ghodsi, M., Salami, M., Zand, N., Kashef, N., \& Larijani, B. (2011). A randomized clinical trial on the effect of low-level laser therapy on chronic diabetic foot wound healing: a preliminary report. Photomedicine Laser Surgery, 29(2):109-14.

Landau, Z., Migdal, M., Lipovsky, A., \& Lubart, R. (2011). Visible light-induced healing of diabetic or venous foot ulcers: a placebo-controlled double-blind study. Photomedicine Laser Surgery, 29(6):399-404.

Leal, E., \& Carvalho, E. (2012). Cicatrização de Feridas: O Fisiológico e o Patológico. Revista Portuguesa de Diabetes, 9(3), $133-43$.

Mathur, R. K., Sahu, K., Saraf, S., Patheja, P., Khan, F., \& Gupta, PK. (2017). Low-level laser therapy as an adjunct to conventional therapy in the treatment of diabetic foot ulcers. Lasers in Medical Science, 32(2):275-82.

Mohajeri-Tehrani, M. R., \& Annabestani, Z. (2014). Effect of low-intensity direct current on expression of vascular endothelial growth factor and nitric oxide in diabetic foot ulcers. Journal of Rehabilitation Research \& Development, 51(5), 815-24.

Moura, R. O., Nunes, L. C. C., Carvalho, M. E. I. M., \& Miranda, B. R. (2014). Efeitos da luz emitida por diodos (LED) e dos compostos de quitosana na cicatrização de feridas Revisão Sistemática. Journal Of Basic and Applied Pharmaceutical Sciences, 35(4), $513-518$.

Ortíz, M. C. S., Villabona, E. H., Lemos, D. M. C., \& Castellanos, R. (2014). Effects of low level laser therapy and high voltage stimulation on diabetic wound healing. Revista de la Universidad Industrial de Santander, 46(2):107-17.

Peters, M. D. J., Godfrey, C., Mcinerney, P., Munn, Z., Tricco, A. C., Khalil, H. Chapter 11: Scoping Reviews (2020 version). In: Aromataris, E., Munn, Z. (Editors). (2020). JBI Manual for Evidence Synthesis, Joanna Briggs Institute, <https://synthesismanual.jbi.global>.

Polak, A., Kloth, L. C., Blaszczak, E., Taradaj, J., Nawrat-Szoltysik, A., Ickowicz, T., Hordynska, E., Franek, A., \& Kucio, C. (2017). The efficacy of pressure ulcer treatment with cathodal and cathodal-anodal high-voltage monophasic pulsed current: a prospective, randomized, controlled clinical trial. Physical Therapy, 97(8):777-89.

Polak, A., Kloth, L. C., Blaszczak, E., Taradaj, J., Nawrat-Szoltysik, A., Walczak, A., Bialek, L., Paczula, M., Franek, A., \& Kucio, C. (2016). Evaluation of the healing progress of pressure ulcers treated with cathodal high-voltage monophasic pulsed current: results of a prospective, double-blind, randomized clinical trial. Advances in Skin \& Wound Care, 29(10):447-59.

Polak, A., Kucio, C., Kloth, L. C., Paczula, M., Hordynska, E., Ickowicz, T., Blaszczak, E., Kucio, E., Oleszczyk, K., Ficek, K., \& Franek, A. (2018). A Randomized, Controlled Clinical Study to Assess the Effect of Anodal and Cathodal Electrical Stimulation on Periwound Skin Blood Flow and Pressure Ulcer Size Reduction in Persons with Neurological Injuries. Ostomy Wound Management, 64(2), 10-29.

Polak, A., Taradaj, J., Nawrat-Szoltysik, A., Stania, M., Dolibog, P., Blaszczak, E., Zarzeczny, R., Juras, G., Franek, A., \& Kucio, C. (2016). Reduction of pressure ulcer size with high-voltage pulsed current and high-frequency ultrasound: a randomised trial. Journal of Wound Care, 25(12):742-54.

Rosa, S., Rosa, M. F. F., Marques, M. P., Guimarães, G. A., Motta, B. C., Macedo, Y. C. L., Inazawa, P., Dominguez, A., Macedo, F. S., Lopes, C. A. P., \& Rocha, A. F. (2019). Regeneration of diabetic foot ulcers based on therapy with red LED light and a natural latex biomembrane. Annals of Biomedical Engineering, 47(4), 1153-64.

Ruh, A. C., Frigo, L., Cavalcanti, M. F. X. B., Svidnicki, P., Vicari, V. N., Lopes-Martins, R. A. B., Junior, E. C. P. L., Isla, N., Diomede, F. Trubiani, O., \& Fevero, G. M. (2018). Laser photobiomodulation in pressure ulcer healing of human diabetic patients: gene expression analysis of inflammatory biochemical markers. Lasers in Medical Science, 33(1), 165-71. 
Research, Society and Development, v. 10, n. 6, e45910616003, 2021

(CC BY 4.0) | ISSN 2525-3409 | DOI: http://dx.doi.org/10.33448/rsd-v10i6.16003

Santos, J. A. F., Campelo, M. B. D., Oliveira, R. A., Nicolau, R. A., Rezende, V. E. A., \& Arisawa, E. Â. L. (2018). Effects of Low-Power Light Therapy on the Tissue Repair Process of Chronic Wounds in Diabetic Feet. Photomedicine and Laser Surgery, 36(6), 298-304.

Shiwa, S. R., Costa, L. O. P., Moser, A. D. L., Aguiar, I. C., \& Oliveira, L. V. F. (2011). PEDro: a base de dados de evidências em fisioterapia. Fisioterapia em Movimento, 24(3)

Silva, F. M., Moreira, L. S., Silva, M. S., Rodrigues, W., \& Rosa, S. S. R. F. (2019). Uso de Fototerapia para cicatrizaçao de feridas de pés diabéticos. Revista Eletrônica do Programa de Mestrado em Direitos Humanos, 27(Especial), 7-27.

Souza, A. C. S., Costa, M. M. L., Costa, P. H. V., \& Silva, F. S. (2018). Efeito da estimulação elétrica de alta voltagem para o tratamento de úlceras por pressão: um estudo experimental de caso único. Fisioterapia Brasil, 18(6), 676-85.

Tricco, A. C., Lillie, E., Zarin, W., O’Brien, K. K., Colquhoun, H., Levac, D., et al. (2018). PRISMA extension for scoping reviews (PRISMA-ScR): checklist and explanation. Annals of Internal Medicine, 169(7), 467-73.

Vitoriano, N. A. M., Mont'Alverne, D. G. B., Martins, M. I. S., Silva, P. S., Martins, C. A., Teixeira, H. D., Miranda, C. B., Bezerra, L. M. M., Montenegro, R. M., \& Tatmatsu-Rocha, J. C. (2019). Comparative study on laser and LED influence on tissue repair and improvement of neuropathic symptoms during the treatment of diabetic ulcers. Lasers in Medical Science, 34(7), 1365-71. 